\title{
Superharmonic instability, homoclinic torus bifurcation and water-wave breaking
}

\author{
By THOMAS J. BRIDGES \\ Department of Mathematics, University of Surrey, Guildford, Surrey, GU2 7XH, UK
}

(Received 24 October 2003 and in revised form 2 February 2004)

The superharmonic instability is pervasive in large-amplitude water-wave problems and numerical simulations have predicted a close connection between it and crest instabilities and wave breaking. In this paper we present a nonlinear theory, which is a generic nonlinear consequence of superharmonic instability. The theory predicts the nonlinear behaviour witnessed in numerics, and gives new information about the nonlinear structure of large-amplitude water waves, including a mechanism for noisy wave breaking.

\section{Superharmonic instability of water waves}

The superharmonic ( $\mathrm{SH}$ ) instability of travelling surface waves is one of the two principal instabilities of nonlinear periodic travelling waves of finite amplitude, the other being the Benjamin-Feir or modulational instability. The SH instability arises when the momentum of the wave, considered as a function of the wave speed, passes through a critical point. Figure 1 shows a typical momentum-wave speed $(I-c)$ diagram where the first critical point is a maximum.

The SH instability differs from the Benjamin-Feir instablility in that the SH perturbation has the same wavelength as the basic periodic travelling wave. The SH instability is pervasive in water-wave problems (e.g. Longuet-Higgins 1978; Saffman 1985; Tanaka 1985; Tanaka et al. 1987; Jillians 1989; Longuet-Higgins \& Dommermuth 1997), in interfacial wave problems (Holyer 1979), and the KelvinHelmholtz instability (Drazin 1970; Benjamin \& Bridges 1997, § 2.4).

Linear SH instability of water waves was first found by Longuet-Higgins (1978) by computing the eigenvalues of the linearization about periodic travelling waves. Subsequently Tanaka (1985) discovered, in further numerical calculations, that the linear SH instability of water waves is associated with a maximum of the momentum (equivalently, the energy) when considered as a function of the wave speed. In a seminal paper, the structure of the linear problem near an SH instability was illuminated by Saffman (1985). He showed analytically that a momentum (equivalently, energy) maximum corresponds precisely to a change in $\mathrm{SH}$ instability. Using Saffman's Theorem, one can predict a point of SH instability transition (generically) by studying the critical points of the $I-c$ diagram.

One of the most interesting consequences of the $\mathrm{SH}$ instability is that numerical simulations show that SH instability is closely associated with a form of wave breaking (Tanaka et al. 1987; Jillians 1989; Longuet-Higgins \& Dommermuth 1997).

Existing theory for SH instability is however restricted to linearized instability. The only nonlinear results in the literature are obtained by numerical simulation. 


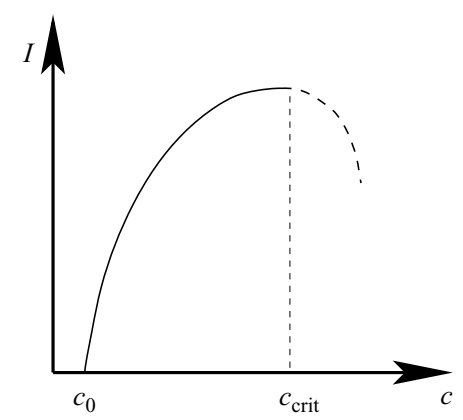

FIgURE 1. A schematic of the momentum-wave speed $(I-c)$ plane for water waves. A superharmonic instability transition arises at the maximum.

In this paper a theory for the nonlinear behaviour near the SH instability transition is presented. The theory is based on an application of dynamical systems theory. The main result is that the nonlinear problem near the SH instability always (generically) leads to a homoclinic torus bifurcation. This homoclinic bifurcation explains the results seen in numerical simulations near the SH instability (Tanaka et al. 1987; LonguetHiggins \& Dommermuth 1997).

The ocean environment will have naturally occurring forcing and dissipation. And, it is well known that homoclinic orbits with small damping and forcing can generate complex dynamics. So, with a perturbation (for example, a periodic pressure variation over the ocean), and small damping, the homoclinic torus bifurcation can give rise to complex dynamics which will influence wave breaking, creating a form of wave breaking perturbed by weak chaos, which we will call noisy wave breaking.

So far, this discussion has been limited to the behaviour near the first maximum of the momentum as in figure 1. However, there are further results in the literature about the $I-c$ diagram that suggest a far more dramatic sequence of homoclinic bifurcations. The first maximum in the $I-c$ diagram as shown in figure 1 was found by Longuet-Higgins (1975). Subsequent analysis using matched asymptotic expansions by Longuet-Higgins \& Fox (1978) showed that in the limit as the highest wave is approached the number of critical points of the $I-c$ diagram approaches infinity. Numerical results of Chandler \& Graham (1993) also provide evidence that the number of critical points of the $I-c$ diagram is increasing without bound. In other words, the continuation of the diagram in figure 1 is a spiral with a finite limit $\left(I_{\infty}, c_{\infty}\right)$, and the asymptotics of Longuet-Higgins \& Fox (1978) predict that the spiral is logarithmic.

These results are based on the properties of the Stokes wave. At each critical point of the $I-c$ curve, Saffman's Theorem can be applied to conclude that a pair of eigenvalues, in the linearization about the Stokes wave, is coalescing at the origin. Indeed, Longuet-Higgins \& Tanaka (1997) have computed eigenvalues of the linearization about Stokes waves near the first two critical points (a maximum and minimum) and show explicitly that unstable eigenvalues are generated at both.

The nonlinear theory of this paper extends Saffman's theory into the nonlinear regime. Therefore, one can deduce that near each critical point of the $I-c$ curve, there will be an attendant homoclinic torus bifurcation, and if this sequence is infinite the physical and mathematical consequences are manifold.

The existence of a homoclinic torus bifurcation for water waves near an $\mathrm{SH}$ instability follows from four observations. (a) Numerical results as noted above show 
that the $I-c$ diagram for periodic Stokes waves has at least one critical point and possibly an infinite number. (b) Saffman's theorem shows that critical points in the $I-c$ plane imply a coalescence of two eigenvalues at zero. (c) Hamiltonian theory (using the fact that the water-wave problem is Hamiltonian) shows that locally in the nonlinear problem near this coalescence, there is a homoclinic bifurcation (cf. Arnold, Kozlov \& Neishtadt 1993, pp. 264 and 270). (d) Travelling waves are not equilibria but relative equilibria and so there is an attendant circle symmetry. Using this symmetry it follows that there is a toral surface of homoclinic bifurcations.

This combination of theory is formal, but can be made precise using Birkhoff normal form theory for water waves (see Craig 1996 for an exposition on normal form theory for water waves). However, the important feature of the above observations is that the nonlinear consequences of SH instability can be predicted a priori, before explicit computation or numerical simulation.

Normal form theory shows that there is a four-dimensional subspace of the infinitedimensional water-wave problem in which the principal dynamics occurs. Therefore, a simplified model problem - which has all the features of the reduced four-dimensional subspace associated with the water-wave problem near an SH instability - will be used to introduce the main features of the connection between SH instability and homoclinic torus bifurcation. Then the implications for water waves are discussed.

\section{A model for nonlinear SH instability}

A simplified model, which has all the essential geometric features of a Stokes wave near an energy-momentum maximum and a change of $\mathrm{SH}$ instability, is

$$
A_{t t}-2 \mathrm{i} a A_{t}-r A-|A|^{2} A=0, \quad A(t) \in \mathbb{C},
$$

with $a, r \in \mathbb{R}$ with $a>0$ and $0<r<a^{2}$. Although this model is not derived explicitly from water waves, it is in fact the model obtained when the SH instability arises at low amplitude, as in the Kelvin-Helmholtz problem (cf. Drazin 1970; Benjamin \& Bridges 1997, § 2.4). Indeed, one of the consequences of the observations in this paper is that a homoclinic torus bifurcation is also a central feature of the formation of nonlinear Kelvin-Helmholtz billows (the full implications of this observation will be considered elsewhere).

The energy $H$ and momentum $I$ for (2.1) are

$$
H=\left|A_{t}\right|^{2}-r|A|^{2}-\frac{1}{2}|A|^{4}, \quad I=a|A|^{2}-\operatorname{Im}\left(\bar{A} A_{t}\right) .
$$

They satisfy $I_{t}=H_{t}=0$ for all time and model the energy and momentum of water waves. The periodic Stokes wave in the model (2.1) is represented by $A(t)=A_{0} \mathrm{e}^{\mathrm{i} c t}$. Substitution of this form into (2.1) results in the nonlinear dispersion relation

$$
(c-a)^{2}+\left|A_{0}\right|^{2}=a^{2}-r,
$$

and the momentum along this branch is $I=(a-c)\left|A_{0}\right|^{2}$. Let $c_{0}=a-\sqrt{a^{2}-r}$. Then for $c_{0}<c<a$ the wave speed $c$ is a positive increasing function of amplitude. The slope of the momentum is positive for $c_{0}<c<c_{\text {crit }}$ where

$$
c_{\text {crit }}=a-\sqrt{\frac{1}{3}\left(a^{2}-r\right)}<a .
$$

At $c_{\text {crit }}$ there is a change in slope. Before considering the linear and nonlinear behaviour near $c_{\text {crit }}$, it will be of value to view this problem from a Hamiltonian perspective. 


\subsection{Hamiltonian structure of the SH instability}

As in the linear theory of Saffman for the SH instability, the Hamiltonian structure turns out to be fundamental in the analysis of the nonlinear SH problem as well. In this subsection, a Hamiltonian analysis of the SH instability of the model problem (2.1) is presented.

Let $(q, p) \in \mathbb{R}^{4}$ be real coordinates defined by $q_{1}+\mathrm{i} q_{2}=A$ and $p_{1}+\mathrm{i} p_{2}=A_{t}-\mathrm{i} a A$; then the model equation (2.1) can be written in the standard Hamiltonian form

$$
\boldsymbol{J} U_{t}=\nabla H(U), \quad U=(q, p) \in \mathbb{R}^{4}, \quad \boldsymbol{J}=\left[\begin{array}{rr}
\mathbf{0} & -\boldsymbol{I} \\
\boldsymbol{I} & \mathbf{0}
\end{array}\right],
$$

where $\boldsymbol{I}$ is the identity on $\mathbb{R}^{2}$, and

$$
H(U)=\frac{1}{2}\left(p_{1}^{2}+p_{2}^{2}\right)-a\left(p_{1} q_{2}-p_{2} q_{1}\right)-\frac{1}{2}\left(r-a^{2}\right)\left(q_{1}^{2}+q_{2}^{2}\right)-\frac{1}{4}\left(q_{1}^{2}+q_{2}^{2}\right)^{2},
$$

and the momentum in these coordinates is $I=p_{1} q_{2}-p_{2} q_{1}$.

The system (2.5) is invariant with respect to the group $\mathrm{SO}(2)$ with action $\mathrm{G}_{\theta}=$ $\left(\mathrm{R}_{\theta} q, \mathrm{R}_{\theta} p\right)$ where $\mathrm{R}_{\theta}$ is the standard action of $\mathrm{SO}(2)$ on $\mathbb{R}^{2}$. This $\mathrm{SO}(2)$ symmetry in the model problem is analogous to the translation invariance of the water-wave problem restricted to periodic functions. The details of how $\mathrm{SO}(2)$ symmetry arises in the spatially periodic water-wave problem are given in $\$ 2.2$ of Benjamin \& Bridges (1997).

The basic state (representing the Stokes wave) can be characterized as a relative equilibrium associated with the group $\mathrm{SO}(2)$

$$
U(t)=\mathrm{G}_{\theta(t)} U_{0}, \quad \theta(t)=c t+\theta_{0},
$$

with $U_{0} \in \mathbb{R}^{4}$ satisfying $\nabla H\left(U_{0}\right)+c \nabla I\left(U_{0}\right)=0$. Explicit calculations show that

$$
U_{0}=\left(q_{0}, c \boldsymbol{J}_{2} q_{0}\right) \quad \text { with } \quad\left|q_{0}\right|^{2}=a^{2}-r-(c-a)^{2}, \quad \boldsymbol{J}_{2}=\left[\begin{array}{rr}
0 & -1 \\
1 & 0
\end{array}\right] .
$$

This result is a symplectic formulation of the basic state $A(t)=A_{0} \mathrm{e}^{\mathrm{i} c t}$ of (2.1). Formulating the basic state as in (2.7) so that $U_{0}$ is a critical point of the energy restricted to level sets of the momentum is analogous to the construction of travelling waves of the water-wave problem as relative equilibria (cf. Benjamin \& Bridges 1997, $\S 2)$.

Substituting the solution (2.7) into the energy and momentum we find

$$
H_{0}(c)=\frac{1}{4}\left|q_{0}\right|^{2}\left(3 c^{2}-2 a c-r\right), \quad I_{0}(c)=(a-c)\left|q_{0}\right|^{2},
$$

with derivatives $I_{0}^{\prime}(c)=-\left(a^{2}-r\right)+3(c-a)^{2}=-\frac{1}{c} H_{0}^{\prime}(c)$. Note that when $c \neq 0$, the derivative $I_{0}^{\prime}(c)=0$ if and only if $H_{0}^{\prime}(c)=0$. This result is a general property of periodic travelling waves characterized by a constrained variational principle (cf. Benjamin \& Bridges 1997, §3).

The association between the energy-momentum maximum and the SH instability is elementary to establish for this model. Linearize (2.5) about (2.7) and look for solutions proportional to $\mathrm{e}^{\lambda t}$. Then the characteristic equation is

$$
\operatorname{det}\left[D^{2} H\left(U_{0}\right)-c D^{2} I\left(U_{0}\right)-\lambda \boldsymbol{J}\right]=\lambda^{4}+2 I_{0}^{\prime}(c) \lambda^{2}=0 .
$$

Figure 2 shows the behaviour of the eigenvalues of the linear stability problem as $I_{0}(c)$ passes through a maximum. 


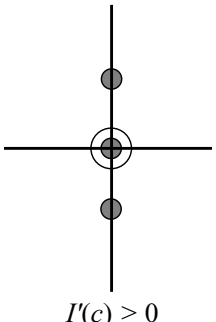

$I^{\prime}(c)>0$

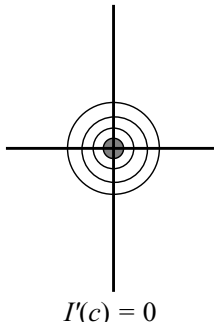

$I^{\prime}(c)=0$

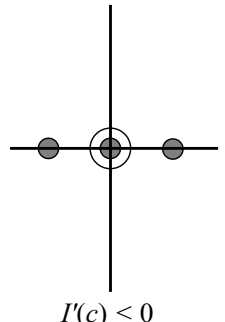

$I^{\prime}(c)<0$

FIGURE 2. A schematic of the eigenvalue movement of the linearization of (2.5) about the basic state (2.7), when $I^{\prime}(c)$ changes sign.

\section{Homoclinic torus bifurcation}

Using the model system, it will be shown explicitly that $\mathrm{SH}$ instability always leads to a homoclinic torus bifurcation. Then it is shown that this nonlinear bifurcation is a generic property of any Hamiltonian system with a state that has an energymomentum maximum of the form shown in figure 1.

Introduce the following change of coordinates, $\left(q_{1}, q_{2}, p_{1}, p_{2}\right) \rightarrow(\xi, \eta, \theta, I)$ :

$$
\left.\begin{array}{ll}
q_{1}=\xi \cos \theta, & q_{2}=\xi \sin \theta, \\
p_{1}=\eta \cos \theta+I \sin \theta / \xi, & p_{2}=\eta \sin \theta-I \cos \theta / \xi .
\end{array}\right\}
$$

In these coordinates the symplectic structure remains the same, and the governing equations are mapped to

$$
\begin{aligned}
& \dot{\xi}=\eta, \\
& \dot{\eta}=\left(r-a^{2}\right) \xi+\xi^{3}+I^{2} / \xi^{3}, \\
& \dot{\theta}=-I / \xi^{2}+a, \\
& \dot{I}=0 .
\end{aligned}
$$

The system (3.2) provides natural coordinates to split the phase space and study the dynamics near the basic state (2.7). Equations $(3.2 c, d)$ here give the dynamics tangent to the basic state, and $(3.2 a, b)$ give the dynamics normal to the basic state.

Now, let $\left(\xi_{0}, \eta_{0}, \theta_{0}, I_{0}\right)$ be a basic state of the form (2.7) in the coordinates (3.1):

$$
\theta_{0}=c t, \quad \eta_{0}=0, \quad \xi_{0}^{2}=a^{2}-r-(c-a)^{2},
$$

and $I=I_{0}(c)$, and introduce perturbed coordinates: $\xi=\xi_{0}+u, \eta=\eta_{0}+v$, and $\theta=c t+\phi$ with $I-I_{0}$ small. Then, expanding the nonlinear differential equation (3.2) in a Taylor series about the basic state leads to the system

$$
\begin{aligned}
\dot{u} & =v, \\
\dot{v} & =-2 I_{0}^{\prime}(c) u+\frac{2 I_{0}}{\xi_{0}^{3}}\left(I-I_{0}\right)+b(c) u^{2}+\cdots, \\
\dot{\phi} & =-\frac{1}{\xi_{0}^{2}}\left(I-I_{0}\right)+\frac{2 I_{0}}{\xi_{0}^{3}} u+\cdots, \\
\dot{I} & =0
\end{aligned}
$$

where $b(c)=\left(3 / \xi_{0}\right)\left(a^{2}-r+(c-a)^{2}\right)>0$. Now this system is valid in the neighbourhood of any value of momentum level set $I_{0}$ along the $(I, c)$ curve in figure 1 . However, $I_{0}^{\prime}(c)=0$ (or $I_{0}^{\prime}(c) \approx 0$ ) at (or very near) the maximum of the curve in which case the 


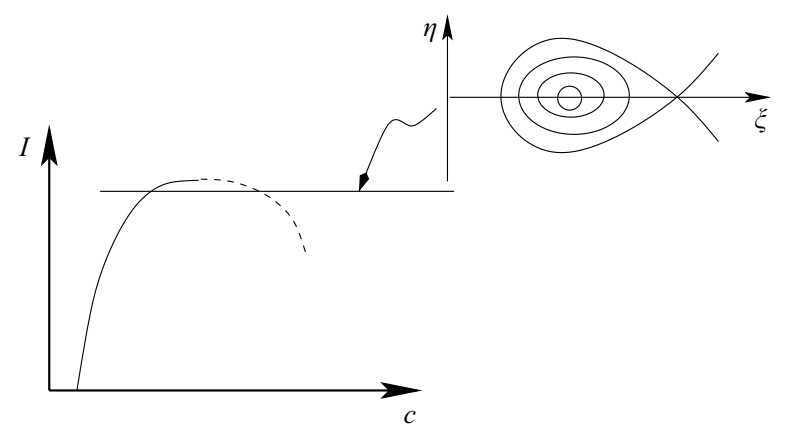

FIGURE 3. A section of the phase-space structure when $I \approx I_{\text {crit }}$ but $I_{\text {crit }}\left(I-I_{\text {crit }}\right)<0$.

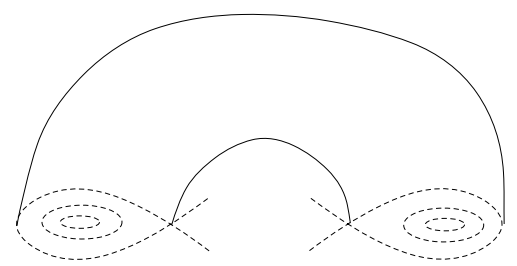

FIGURE 4. A schematic of the homoclinic torus.

reduced system $(3.3 a, b)$ becomes

$$
\begin{aligned}
& \dot{u}=v, \\
& \dot{v}=\frac{2 I_{\text {crit }}}{\xi_{0}^{3}}\left(I-I_{\text {crit }}\right)+b\left(c_{\text {crit }}\right) u^{2}+\cdots .
\end{aligned}
$$

This reduced system has two fixed points (which represent periodic solutions in the original problem), one of which is elliptic and the other hyperbolic, for $I_{\text {crit }}\left(I-I_{\text {crit }}\right)<0$. The stable and unstable manifolds of the hyperbolic point coincide resulting in a homoclinic connection. This is shown schematically in the upper part of figure 3 . It is a homoclinic torus, because the trajectory winds around the torus with $(3.3 c)$ as shown schematically in figure 4 .

\subsection{Homoclinic torus bifurcation of water waves}

Although the above theory has been presented for the model problem, the theory carries over to water waves. Water waves can be modelled as an infinite-dimensional Hamiltonian system using the Zakharov (1968) formulation with canonical variables $(\eta, \Phi)$ where $\eta(x, t)$ is the free-surface elevation and $\Phi(x, t)$ is the velocity potential evaluated at the free surface, and

$$
\eta_{t}=\frac{\delta H}{\delta \Phi}, \quad \Phi_{t}=-\frac{\delta H}{\delta \eta} .
$$

Let $\eta(x, t)=\widehat{\eta}(x-c t)+\widetilde{\eta}(x, t)$ and $\Phi(x, t)=\widehat{\Phi}(x-c t)+\widetilde{\Phi}(x, t)$ where $(\widehat{\eta}, \widehat{\Phi})$ represents the Stokes wave. The perturbed variables $(\widetilde{\eta}, \widetilde{\Phi})$ will be governed by a Hamiltonian system. By choosing new coordinates and performing normal form transformations 
the perturbed water-wave problem can be transformed to

$$
\begin{aligned}
\dot{\xi} & =a_{1} \eta+\cdots \\
\dot{\eta} & =-a_{2} I_{0}^{\prime}(c)\left(\xi-\xi_{0}\right)+a_{3}\left(I-I_{0}\right)+b(c)\left(\xi-\xi_{0}\right)^{2}+\cdots, \\
\dot{\theta} & =a_{4}\left(I-I_{0}\right)+a_{5}\left(\xi-\xi_{0}\right)+\cdots, \\
\dot{I} & =0 \\
\dot{Z} & =f(Z, \xi, \eta, \theta, I)
\end{aligned}
$$

where $Z$ represents the infinite-dimensional set of complementary variables for water waves. The coefficient $a_{1}$ can be scaled to be \pm 1 . The other coefficients $a_{2}, a_{3}, b(c), a_{4}$ and $a_{5}$ are generically non-zero. They can be computed from information associated with a given travelling wave and using Birkhoff normal form theory. However, more importantly, the conclusion of homoclinic torus bifurcation occurs regardless of the specific values of these coefficients.

By formally truncating the above system, there is a four-dimensional subspace on which the dynamics is reduced to the local analysis of the model equation in (3.3), with the attendant homoclinic torus bifurcation. Indeed, because of the additional purely imaginary eigenvalues, the dynamics will be in general more complex than just a homoclinic bifurcation. See for example the work of Broer et al. (1993), where the case of a homoclinic bifurcation with one extra pair of purely imaginary eigenvalues is studied and shown to have more complex dynamics than an isolated homoclinic bifurcation.

The fact that any system with an energy-momentum maximum can be transformed to the form (3.6) is based on three observations. First, any symmetry-based solution which has a maximum of the energy-momentum has a pair of eigenvalues (of the linear stability problem) passing through zero - this is Saffman's Theorem for SH instability. Secondly, any Hamiltonian system with a pair of eigenvalues passing through the origin always has a homoclinic bifurcation (see $\$ 4$ of Chapter 7 in Arnold et al. 1993 and Broer et al. 1993) as long as the coefficient of the first nonlinear term (i.e. the coefficient $b(c)$ in (3.3)) is non-zero. Thirdly, in the symmetric context (such as the model problem and the water-wave problem) the homoclinic orbit has an extra dimension, resulting in a homoclinic torus.

\section{Breaking of water waves}

While the model (2.1) shows the local nonlinear behaviour near a transition of $\mathrm{SH}$ instability, we need the full splendor of the water-wave equations to see the consequences for wave breaking.

The main results in the literature we can appeal to for comparison are the numerical simulations of the nonlinear problem by Longuet-Higgins \& Dommermuth (1997) and Jillians (1989). Tanaka et al. (1987) also show numerical simulations near SH instability but the basic state is a solitary wave there. All the numerical simulations show that certain initial conditions lead to a form of wave breaking as shown schematically in figure 5. The other possibility witnessed in numerics is an evolution to a slower periodic wave (either in a recurrent or attractive way). Both of these numerical observations are predicted by the homoclinic torus bifurcation. If perturbations are to the left side of the hyperbolic point in the reduced two-dimensional phase space (i.e. to the left of the hyperbolic periodic point in the $\xi-\eta$ phase space in figure 3) they will circle around the slower periodic wave. However, the numerical solutions have a small amount of numerical dissipation (see discussion in Dold 1992). Therefore the 


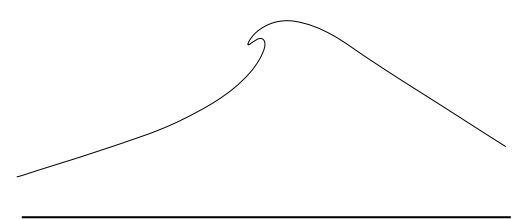

FIGURE 5. A schematic based on the numerical experiments of Longuet-Higgins \& Dommermuth (1997) depicting the development of a wave after a superharmonic instability.

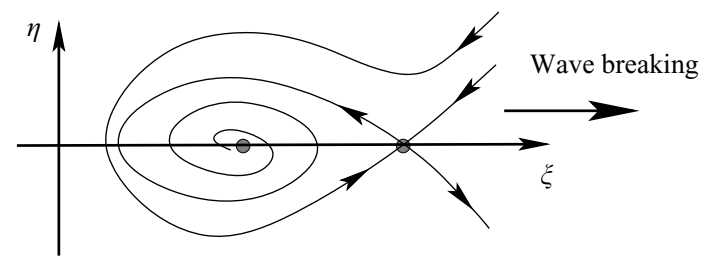

FIGURE 6. A schematic of the reduced phase space when weak damping is included.

elliptic periodic orbit will develop an attracting direction, and so the behaviour in the numerical solutions is probably closer to the (reduced) phase space schematic shown in figure 6. Depending on the amount of damping, the initial conditions are either attracted to the slower periodic wave or they will circle it in a recurrent fashion, slowly settling on the slower periodic wave eventually. This behaviour is in agreement with the numerical results.

To the right of the hyperbolic periodic point in the $\xi-\eta$ phase space in figure 3 there are no other periodic solutions locally, and so trajectories escape. It is clear from the numerical simulations that this direction leads to wave breaking. Since wave breaking is an unsteady solution, it suggests that there are indeed no other steady solutions nearby. The only known steady solution in this regime is the steady breaking of wave Jenkins (1994). However, Jenkins' solution is multi-valued and so would not be seen by the numerics.

\subsection{A potentially infinite sequence of homoclinic torus bifurcations}

The theory that predicts a homoclinic torus bifurcation relies only on the existence of a critical point of the momentum in the $I-c$ diagram. As noted in the introduction, numerical results of Longuet-Higgins (1975); Chandler \& Graham (1993) and the asymptotics of Longuet-Higgins \& Fox (1978) for the $I-c$ diagram and the numerical calculations of the linear stability problem of Longuet-Higgins \& Tanaka (1997) all show that there is more than one point of SH instability transition, and potentially an infinite number.

A schematic of the $I-c$ diagram for water waves based on these results is shown in figure 7 with the sequential SH points marked. It is apparent from the theory in this paper that each of the SH transition points will correspond generically to points of homoclinic torus bifurcation, and this is also shown schematically in figure 7 . One can conjecture a far more dramatic sequence of bifurcations based on the theory of Longuet-Higgins \& Fox (1978). Using matched asymptotic expansions, LonguetHiggins \& Fox (1978) show that as the highest wave is approached the $I-c$ curve spirals infinitely often. For Stokes gravity waves in deep water, Longuet-Higgins \& Fox (1978) found the asymptotic form of the phase speed $c$ and the momentum $I$ to be

$$
c^{2}=c_{\infty}^{2}+A \epsilon^{3} \cos (3 \mu \ln \epsilon+B), \quad I=I_{\infty}+A^{\prime} \epsilon^{3} \cos \left(3 \mu \ln \epsilon+B^{\prime}\right),
$$




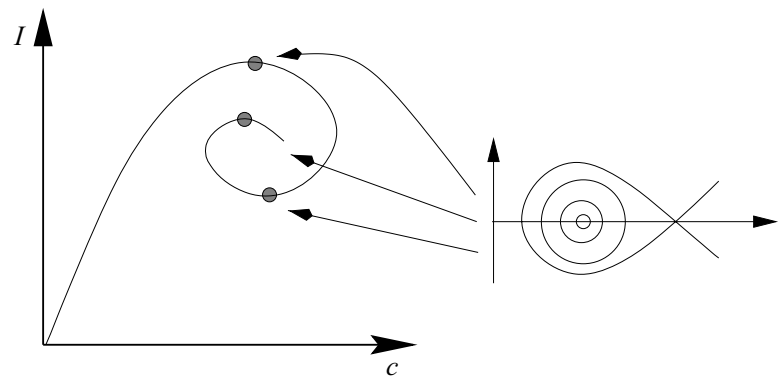

FiguRE 7. A schematic of the momentum-wave speed $(I-c)$ plane showing a potential sequence of $\mathrm{SH}$ instability points and potential homoclinic bifurcations for periodic travelling water waves.

where $c_{\infty}$ and $I_{\infty}$ are the speed and momentum of the highest wave and expressions for the order-one constants $A, A^{\prime}, \mu, B$ and $B^{\prime}$ are given in the paper. Here, $\epsilon$ is a small parameter tending to zero as the limiting wave is approached. As $\epsilon \rightarrow 0$, $\ln \epsilon \rightarrow-\infty$ leading to an infinite spiral in the $I-c$ plane.

At every critical point of the momentum as a function of the wave speed a pair of eigenvalues coalesces in the linear stability problem. By the theory in this paper there is an attendant homoclinic torus bifurcation. Therefore we can reasonably conjecture that as the highest Stokes wave is approached, the phase space is being filled out by homoclinic bifurcations. On the physical side, this is of interest as it gives a geometric structure of the phase space of the nonlinear problem near the highest Stokes wave, and theoretically the sequence of homoclinic bifurcations is of interest as a fundamental new bifurcation in symmetric Hamiltonian sytems in infinite dimension - especially if the sequence of homoclinic bifurcations is infinite.

\subsection{Noisy wave breaking}

It is well known in dynamical systems theory that the addition of small damping and forcing to an ODE with a homoclinic orbit can lead to complex dynamics, i.e. 'horseshoe chaos' (Guckenheimer \& Holmes 1990). The existence of horseshoe chaos in the model problem (2.5), perturbed by damping and forcing, could be proved using analytical methods. However, an open question is the implications of this form of weak chaos on the form of the breaking waves induced by SH instability. The physical implications of horseshoe chaos on SH-induced wave breaking could be tested by modifying existing numerical algorithms in the literature, such as the algorithm in Longuet-Higgins \& Dommermuth (1997), by adding weak damping and explicit forcing.

\section{REFERENCES}

Arnold, V. I., Kozlov, V. V. \& Neishtadt, A. I. 1993 Mathematical aspects of classical and celestial mechanics. Dynamical Systems III, Encycl. Math. Sci., Vol. 3. Springer.

Benjamin, T. B. \& BRidges, T. J. 1997 Reappraisal of the Kelvin-Helmholtz problem. Part 2. Interaction of the Kelvin-Helmholtz, Superharmonic and Benjamin-Feir instabilities. J. Fluid Mech. 333, 327-373.

Broer, H. W., Chow, S. N., Kim, Y. I. \& Vegter, G. 1993 A normally elliptic Hamiltonian bifurcation. Z. Angew. Math. Phys. 44, 389-432.

Chandler, G. A. \& Graham, I. G. 1993 The computation of water waves modelled by Nekrasov's equation. SIAM J. Numer. Anal. 30, 1041-1065. 
Craig, W. 1996 Birkhoff normal forms for water waves. In Mathematical Problems in the Theory of Water Waves (Luminy, 1995), Contemp. Math. 200, 57-74. Am. Math. Soc: Providence.

Dold, J. W. 1992 An efficient surface-integral algorithm applied to unsteady gravity waves. J. Comput. Phys. 103, 90-115.

Drazin, P. G. 1970 Kelvin-Helmholtz instability of finite amplitude. J. Fluid Mech. 42, 321-335.

Guckenheimer, J. \& Holmes, P. J. 1990 Nonlinear Oscillations, Dynamical Dystems, and Bifurcations of Vector Fields. Springer.

Holyer, J. Y. 1979 Large amplitude progressive interfacial waves. J. Fluid Mech. 93, 433-448.

Jenkins, A. D. 1994 A stationary potential-flow approximation for a breaking-wave crest. J. Fluid Mech. 280, 335-347.

JiLLIANS, W. J. 1989 The superharmonic instability of Stokes waves in deep water. J. Fluid Mech. 204, 563-579.

Longuet-Higgins, M. S. 1975 Integral properties of periodic gravity waves of finite amplitude. Proc. R. Soc. Lond. A 342, 157-174.

Longuet-Higgins, M. S. 1978 The instabilities of gravity waves of finite amplitude in deep water. I. Superharmonics. Proc. R. Soc. Lond. A 360, 471-488.

Longuet-Higgins, M. S. \& Dommermuth, D. G. 1997 Crest instabilities of gravity waves. Part 3. Nonlinear development and breaking. J. Fluid Mech. 336, 33-50.

Longuet-Higgins, M. S. \& Fox, M. J. H. 1978 Theory of the almost-highest wave. Part 2. Matching and analytic extension. J. Fluid Mech. 85, 769-786.

Longuet-Higgins, M. S. \& Tanaka, M. 1997 On the crest instabilities of steep surface waves. J. Fluid Mech. 336, 51-68.

SAFFMAN, P. G. 1985 The superharmonic instability of finite-amplitude water waves. J. Fluid Mech. 159, 169-174.

Tanaka, M. 1985 The stability of steep gravity waves. Part 2. J. Fluid Mech. 156, 281-289.

Tanaka, M., Dold, J. W., Lewy, M. \& Peregrine, D. H. 1987 Instability and breaking of a solitary wave. J. Fluid Mech. 185, 235-248.

Zakharov, V. E. 1968 Stability of periodic waves of finite amplitude on the surface of a deep fluid. Zh. Prikl. Mekh. Fiz. 9, 86-94 (English transl: J. Appl. Mech. Tech. Phys. 2, 190). 